\title{
Le rôle de l'apoptose dans la genèse et le traitement du cancer
}

L'apoptose des cellules tumorales, spontanée ou induite, règle la croissance des tumeurs. Cette " mort cellulaire programmée " est sous la dépendance d'oncogènes dont certains, comme $c$-myc, sont aussi impliqués dans le contrôle de la prolifération, tandis que d'autres, tels que bcl-2, sont essentiellement inhibiteurs de l'apoptose. L'orientation du métabolisme cellulaire vers la mort ou la prolifération pourrait se faire au niveau de pointscontrôle. La p53 semble être un de ces points. La compréhension des mécanismes de l'apoptose dans les cellules tumorales et de son induction par divers traitements ouvre de nouvelles perspectives dans la prophylaxie des tumeurs malignes et leur traitement.

\section{Éric Solary Richard Bertrand Yves Pommier}

\author{
ADRESSE \\ E. Solary : praticien hospitalo-universitaire. \\ Hématologic clinique, CHU Le Bocage, BP \\ 1542, 21034 Dijon Cedex, France. R. Ber- \\ trand : docteur ès sciences. Institut du cancer, \\ Centre hospitalier Notre-Dame, Université \\ de Montréal, 1560 rue Sherbroke Est, Mon- \\ tréal, Québec, Canada H2L4M1. Y. Pom- \\ mier : docteur en médecine, docteur ès sciences. \\ Laboratory of Molecular Pharmacology, \\ DCT, National Institutes of Health, Natio- \\ nal Cancer Institute, Bldg 37, Room 5C27, \\ Bethesda, MD 20892, Etats-Unis.
}

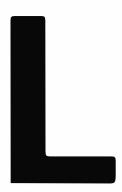

'apoptose est un mode ubiquitaire de mort cellulaire qui jouc un rôle opposé à celui de la mitose dans la régulation des populations cellulaires animales [1]. Elle intervient, par exemple, dans le développement embryonnaire et fæal (depuis la régulation de la cellularité du blastocyste jusqu'à l'organisation du système nerveux central ou de l'appareil génital), dans l'élaboration du système immunitaire et dans l'homéostasie des tissus hormonodépendants. L'homéostasie tissulaire nécessite un équilibre constant entre mort et prolifération cellulaire. Dans les organismes supérieurs, le métabolisme cellulaire est orienté par des signaux (hormones, facteurs de croissance) provenant des cellules voisines : les uns contrôlent sa prolifération, les autres son programme de suicide [2]. Les facteurs de croissance hématopoḯtiques, par exemple, ont pour fonction première de prévenir l'apoptose des cellules capables de les reconnaître [3]. D’une façon générale, le " suicide " cellulaire est activé pour éliminer sélectivement les cellules devenues indésirables. Il peut s'agir de cellules lésécs ou sénescentes (polynucléaires accumulés sur un site inflammatoire), de cellules reconnues comme étrangères ou prénéoplasiques (les cellules cytotoxiques tuent leurs cellules cibles en induisant leur apoptose), de cellules qui ne sont plus à leur place et ont perdu le contact avec leur micro-environnement (cellules épidermiques ayant migré dans le tissu sous-cutané à la suite d'un traumatisme), ou de cellules en excès entrant en compétition avec d'autres cellules vis-à-vis d'un signal inhibiteur [2]. 


\section{RÉFÉRENCES}

1. Kerr JFR, Wyllic AH, Currie AR. Apoptosis : a basic biological phenomenon with wide ranging implications in tissue kinetics. Br J Cancer $1972 ; 26$ : 239-57.

2. Raff MC. Social control on cell survival and cell death. Nature 1992 ; 356 : 397-400

3. Williams GT, Smith CA, Spooncer E, Dexter 'TM, Taylor DR. Hacmopoietic colony stimulating factors promote cell survival by suppressing apoptosis. Nature 1990 ; 343 : 76-8.

4. Arends MJ, Wyllic AH. Apoptosis mechanisms and roles in pathology. Int Rev Exp Pathol 1991; 32 : 223-54.

5. Bursch W, Oberhammer F, SchulteHermann R. Cell death by apoptosis and its protective role against discase. Trends Biol Sci 1992; 13 : 245-51.

6. Groux H, Torpier G, Monte D, Mouton Y, Capron A, Ameisen JC. Activationinduced death by apoptosis in $\mathrm{CD} 4{ }^{+} \mathrm{T}$ cells from human immunodeficiency virus asymptomatic individuals. J Exp Med 1992 ; $175: 331-6$.

7. Watanabe-Fukunaga $\mathrm{R}$, Brannan $\mathrm{CI}$, Copeland NG, Jenkins NA, Nagata S. Lymphoproliferation disorder in mice explained by defects in Fas antigen that mediates apoptosis. Nature 1992 ; 356 : 314-17.

8. Goldstein P, Ojcius DM, Young JDE. Cell death mechanisms and the immune system. Immunol Rev 1991; 121: 29-65.

9. Korsmeyer SJ. Bcl-2 initiates a new category of oncogenes : regulators of cell death. Blood 1992 ; 80 : 879-86

10. Evan GI, Wyllic AH, Gilbert CS, Littlewood ' $\mathrm{DD}$, Land H, Brooks M, Waters CM, Penn LZ, Hancock DC. Induction of apoptosis in fibroblasts by c-myc protein. Cell 1992 ; 69 : 119-28.

11. Lane DP. p53, guardian of the genome. Nature $1992 ; 358$ : 15-6.

12. Goldstein P. Mort programmée et terrain cellulaire. médecine/sciences 1991; 7 : 681-88.

13. Arends MJ, Morris RG, Wyllie AH. Apoptosis : the role of the endonuclease. $\mathrm{Am}$ $J$ Pathol $1990 ; 136$ : 593-608.

14. Collins RJ, Harmon BV, Gobé GC, Kerr JFR. Internucleosomal DNA cleavage should not be the sole criterion for identifying apoptosis. Int Rad Biol $1992 ; 61$ :
Dès lors, on comprend les effets délétères que peut avoir toute dérégulation de l'apoptose, qu'clle soit activće par un signal inhabituel (infection virale, agression chimique ou physique) ou qu'elle soit diminuée par insensibilité aux signaux inducteurs (tératogenèse, auto-immunité, oncogenèse) [4-9]. En envisageant l'oncogenèse comme la consćquence d'une dérégulation de l'apoptose, plusieurs notions nouvelles sont apparues. La première est qu'un oncogène, en l'occurrence $b c l-2$, peut agir non pas en stimulant la prolifération cellulaire (comme c-myc), ou en prévenant l'émergence de clones malins (comme $\mathrm{Rb}$ ou p53), mais en inhibant l'apoptose des cellules dans lesquelles il s'exprime [9]. Plus récemment, l'oncogène $c-m y c$, qui est impliqué dans la prolifération cellulaire en réponse à divers facteurs de croissance, s'est révélé capable d'induire l'apoptose lorsqu'il reste fortement exprimé dans des cellules privées de signal mitogénique [10]. Enfin, la p53, dont la disparition ou l'inactivation est observée dans la plupart des tumeurs malignes, peut orienter la cellule vers l'apoptose ou la prolifération en fonction de divers signaux internes et externes [11]. L'analyse des mécanismes de régulation de l'apoptose ouvre de nouvelles perspectives thérapeutiques dans le domaine du cancer.

\section{Caractéristiques de l'apoptose}

Les caractéristiques de l'apoptose ont fait l'objet de plusieurs articles $(\mathrm{m} / \mathrm{s}$ $n^{\circ} 7$, vol. 7, p. 681, [4, 5, 12]) et ne seront rappelées que brièvement. L'identification d'une cellule apoptotique repose avant tout sur des critères morphologiques stéréotypés dominés par la condensation de la chromatine nucléaire, la fragmentation de la cellule en " corps apoptotiques " et leur phagocytose par les cellules voisines (figure 1). Contrairement à ce que l'on observe dans la nécrose, la chromatine ne flocule pas, les mitochondries ne gonflent pas, la membrane cellulaire n'est pas perméable aux colorants vitaux et l'apoptose ne déclenche pas de réaction inflammatoire car les enzymes lysosomiales ne sont pas relarguées.
La fragmentation internucléosomiale de l'ADN lui donne un aspect caractéristique "en échelle " dans un gel d'électrophorèse. Cette fragmentation est un élément central du processus apoptotique [13]. Son intensité et sa précocité varient suivant les cellules. Elle est cependant inconstante et non spécifique [14]. Typiquement, elle engendre des fragments réguliers qui sont des multiples de 180-200 paires de bases, la longueur d'un fragment d'ADN enroulé autour d'un seul octamère d'histone. Cette fragmentation n'est pas observée dans les cellules nécrotiques. Lors de la nécrose, l'ADN est débarrassé de ses histones par des protéases puis dégradé par des nucléases en fragments dont la taille forme un spectre continu en gel d'électrophorèse.

A l'heure actuelle, aucun marqueur biochimique ou génétique spécifique de l'apoptose n'a été identifié. L'activation d'une ou plusieurs nucléases endogènes est probablement à l'origine des coupures internucléosomiales. Néanmoins, la nature de cette (ou de ces) nucléase(s) reste hypothétique. Le processus apoptotique s'accompagne de modifications caractéristiques de la membrane plasmique, du cytosquelette, des signaux de transduction et du noyau. Ces modifications sont le plus souvent limitées à certains systèmes cellulaires et sont résumées dans le Tableau I (p. 671). La transcription active de gènes non spécifiques et la synthèse de nouvelles protéines ne sont pas toujours nécessaires au déroulement du processus apoptotique [15].

\section{Apoptose et croissance tumorale}

L'apparition puis la croissance d'une tumeur ne sont pas seulement la conséquence d'une prolifération cellulaire excessive. Elles résultent d'un déséquilibre entre prolifération et apoptose. Ce déséquilibre apparaît dès les premières étapes de la carcinogenèse. Il a été étudié au niveau hépatique chez le rat [5]. Un traitement par des composés lipophiles tels que phénobarbital, cyprotérone ou hexachlorocyclohexane induit une hyperplasie hépatique. A l'arrêt du traitement, la régression de l'hyperplasie, plus ou mois rapide en fonction de 
la vitesse d'élimination du mitogène chimique, se fait par apoptose comme la régression des hyperplasics tissulaires physiologiques (utérus après grossesse, glande mammairc après lactation). La reprise du traitement mitogénique inhibe l'apoptose et entretient l'hyperplasie hépatique qui prédispose à la transformation maligne.

Lorsqu'un foyer prénéoplasique hépatique est initié par un carcinogène chimique, le taux de cellules apopto- tiques au scin de cc foyer est plus élevé quc dans le tissu sain environnant. Contrairement aux idées reçues, la phase d'initiation tumorale n'cst donc pas irréversible puisque 80 à $90 \%$ des cellules initiées sont éliminées par apoptose. L'étape de promotion est, ellc aussi, influencée par l'apoptose. Un promoteur tel que le phénobarbital inhibe l'apoptose au niveau des foyers prénéoplasiques et favorise ainsi la croissance tumoralc.
A l'arrĉt du traitement, on observe un taux élcvé de ccllules apoptotiques au scin des foyers prénéoplasiques. La possibilité d'une révcrsion partielle de la promotion tumorale est une notion essentielle dans l'évaluation du risque lié aux promoteurs chimiques. Au sein d'une tumeur, des cellules disparaissent régulièrement par différenciation, desquamation, migration ou mort cellulaire. Le coefficient de perte cellulaire peut être évalué cn

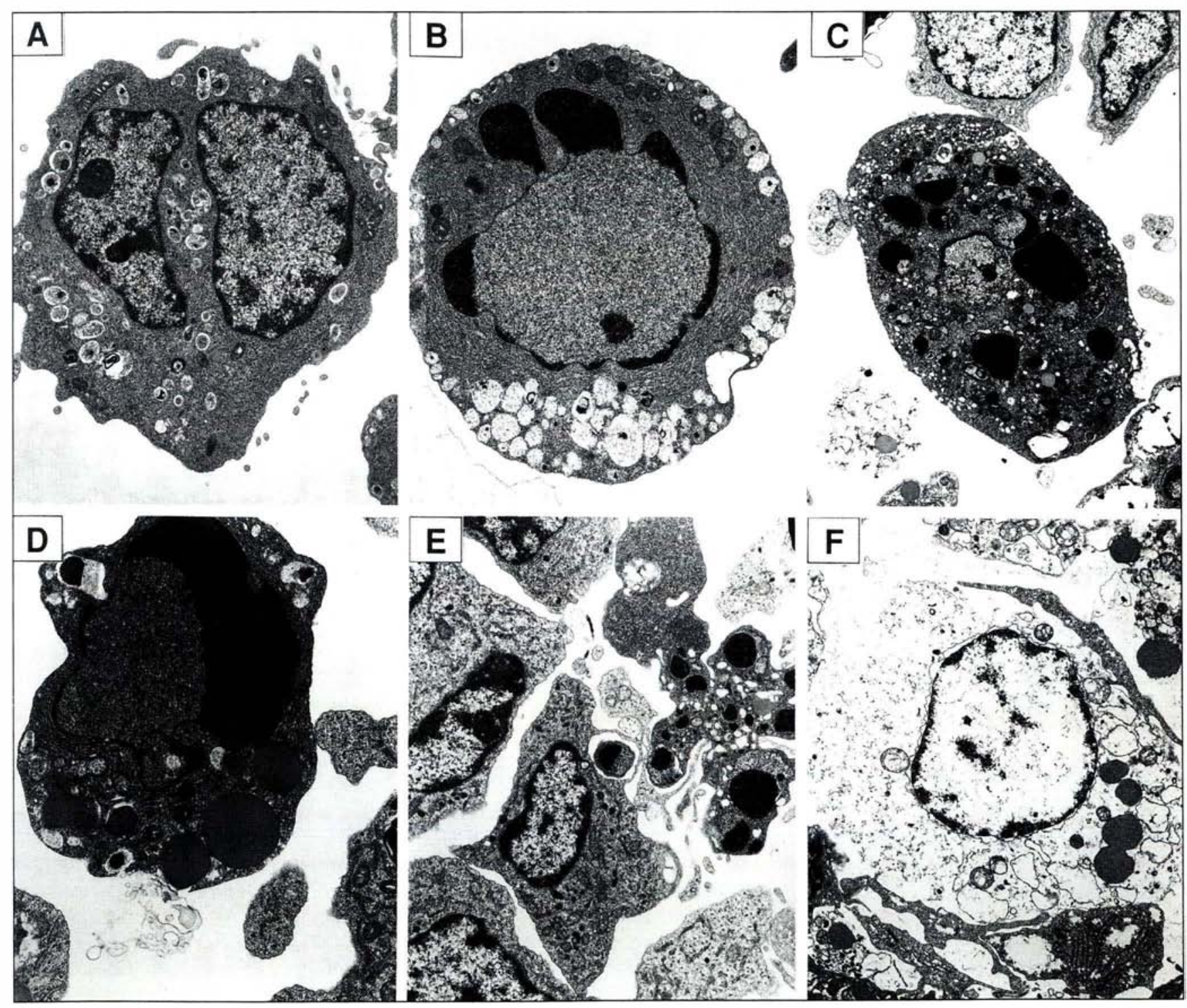

Figure 1. Morphologie de l'apoptose induite par divers agents dans les cellules leucémiques humaines HL-60. A : cellules HL-60 normales (x 6300) B : Première étape : condensation de la chromatine à la périphérie du noyau et condensation du cytoplasme (x 6300). C : fragmentation du noyau (x 4000). D : corps apoptotique renfermant un fragment du noyau et des organelles cytoplasmiques intactes (x 10 000). E : phagocytose des corps apoptotiques (x 6 300). F : nécrose cellulaire post-apoptotique avec rupture de la membrane et chromatine non condensée (x 5 000). (B : camptothécine, $1 \mu \mathrm{M}, 3$ heures ; C,E : acide rétinoïque : $1 \mu \mathrm{M}$ pendant 6 jours; $\boldsymbol{D , F}$ : phorbol myristate acétate $10 \mathrm{nM}$; cellules non adhérentes à 24 heures et 4 jours). 


\section{RÉFÉRENCES}

15. Collins RJ, Harmon BV, Souvlis $\mathrm{T}$, Pope JH, Kerr JFR. Effects of cycloheximide on B-chronic lymphocytic leukaemic and normal lymphocytes in vitro: induction of apoptosis. Br J Cancer 1991 ; 64 : 518-22.

16. Duke RC. Apoptosis in cell-mediated immunity. In : Tomei LD, Cope FO, eds. Apoptosis. The Molecular Basis of Cell Death. Cold Spring Harbor : Cold Spring Harbor Laboratory Press, 1991 : 209-26.

18. Solary E, Bertrand R, Kohn KW, Pommier Y. Differential induction of apoptosis in undifferentiated and differentiated HL-60 cells by DNA topoisomerase I and II inhibitors. Blood 1993; 81 : 1359-68.

19. Yuan J, Horvitz HR. The Caenorhabditis elegans genes ced- 3 and ced- 4 act autonomously to cause programmed ccll death. Dev Biol 1990 ; 138 : 33-41.

20. Clem RJ, Fechheimer M, Miller LK. Prevention of apoptosis by a baculovirus genc during infection of insect cclls. Science 1991 ; 254: 1388-90.

21. Reed JC, Cuddy M, Slabiak T, Croce CM, Nowell PC. Oncogenic potential of bcl-2 demonstrated by gene transfer. Nature 1988 ; 336 : 259-61.

22. Nunez G, London L, Hockenbery D, Alexander M, McKearn JP, Korsmeyer SJ Deregulated bcl-2 gene expression selectively prolongs survival of growth factor-deprived hemopoietic cell lines. I Immunol 1990 ; 144: $3602-10$

23. Hockenbery D, Nunez G, Milliman C, Schreiber RD, Korsmeyer SJ. Bcl-2 is an inner mitochondrial membrane protein that blocks programmed cell death. Nature 1990 ; 348: 334-6.

24. Henderson S, Rowe M, Gregory C, Croom-Carter D, Wang F, Longnecker R, Kieff $\mathrm{E}$, Rickinson A. Induction of bcl-2 expression by Epstein-Barr virus latent membrane protein 1 protects infected B cells from programmed cell death. Cell 1991; 65 : 1107-15.

25. Wyllic AH, Rose KA, Morris RG, Steel CM, Foster E, Spandidos DA. Rodent fibroblast tumours expressing human myc and ras genes: growth, metastasis and endogenous oncogene expression. $\mathrm{Br} J \mathrm{Can}^{-}$ cer $1987 ; 56: 251-9$.

26. Askew DS, Ashmun RA, Simmons BC, Cleveland JL. Constitutive c-myc expression in an IL-3-dependent myeloid cell line suppresses cell cycle arrest and accelerates apo- comparant le temps de doublement des cellules tumorales et le temps de doublement de la masse tumorale. Ce cocfficient cst élcvé dans la plupart des tumeurs. Il peut dépasser $70 \%$ dans les carcinomes colorectaux ou bronchiques et les mélanomes malins [4]. La nécrose et l'apoptose sont les principales causes de perte cellulaire. Des foyers de nécrose sont observés au centre des tumeurs peu vascularisées. Un coefficicnt élevé de perte cellulaire pcut ĉtre observé en l'absence de nécrose : la croissance des carcinomes basocellulaires est lente en l'absence de nécrose et en dépit d'un index mitotique élevé. L'apoptose spontanée des cellules tumorales est observée dans les tumeurs solides de toute taille, en leur périphérie aussi bien qu'en leur centre ainsi que dans les cellules leucémiques fraîches mises en culture Cette apoptose spontanée peut être amplifiée par la thérapeutique, par exemple par la privation hormonale dans les tumeurs hormono-dépendantes.

Les cellules cytotoxiques induiscnt au niveau de leur cible cellulaire des changements morphologiques évoquant l'apoptose. C'est pourquoi le nombre de cellules apoptotiques est particulièrement élevé dans les tumeurs largement infiltrées par des lymphocytes $\mathrm{T}$ cytotoxiques et des cellules NK [4]. Les mécanismes de la mort des cellules cibles sont encore mal expliqués et associent probablement nécrose et apoptose. La frag mentation de l'ADN est très rapide (moins d'une heure), s'associe à des modifications de la chromatine et n'est pas bloquée par les inhibiteurs de la synthèse de macromolécules [12]. Lc rôle des altérations membranaires associées aux interactions cellulaires et des molécules libérées par exocytose (perforine, sérine protéases), dans l'initiation de la fragmentation de l'ADN, reste très discuté [16]. La compréhension des mécanismes de la mort cellulaire induite par les cellules cytotoxiques et de leur modulation devrait permettre d'optimaliser les essais d'immunothérapie actuellement développés.

La différenciation, spontanée ou induite, des cellules tumorales aboutit le plus souvent à la mort cellulaire par apoptose. Une condensation de la chromatine, similaire à celle observée lors du processus apoptotique, est décrite lors de la différenciation de l'épithélium du cristallin ou de la maturation érythrocytaire. La différenciation granulocytaire ou macrophagique des cellules leucémiques humaines HL-60, sous l'influence de divers agents inducteurs, conduit à la mort de ces cellules par apoptose [17]. Paradoxalement, les cellules HL-60, différenciées en macrophages sous l'influence du tpA, deviennent résistantes à l'induction de l'apoptose par divers agents chimiques ou physiques [18]. Ces observations soulignent à quel point l'induction de l'apoptose dépend à la fois du terrain cellulaire, du signal inducteur et de son intensité [12].

\section{Oncogènes} et apoptose

L'existence d'un contrôle génétique de l'apoptose a été démontrée chez le nématode Caenorhabditis elegans avec la mise en évidence de gènes effecteurs (ced 3 et ced 4) et de gènes répresseurs (ced 9) [19]. Chez le baculovirus, un gène répresseur code pour une protéine p35 qui inhibe l'apoptose des cellules infectées [20]. Dans les cellulcs humaines, $b c l-2$ est, à l'heure actuelle, le mieux étudié des gènes dont le produit inhibe l'apoptose $(\mathrm{m} / \mathrm{s}$ $n^{\circ} 1$, vol. 7, p. 88). Cet oncogène a été identifié dans les lymphomes malins folliculaires par clonage de la translocation $\mathrm{t}(14 ; 18)(\mathrm{q} 32 ; \mathrm{q} 21)$. Ce réarrangement chromosomique induit l'hyperexpression de $b c l$-2 en l'amenant au contact du segment $J$ du gène des chaînes lourdes des immunoglobulines (bcl-2/Ig) [9].

Le rôle de $b c l-2$ dans la régulation de la mort ccllulaire est suggéré par plusieurs observations : (1) la mort par apoptose est réduite dans les tumeurs surexprimant $b c l-2$; (2) la transfection de $b c l-2$ dans diverses cellules augmente considérablement leur durée de vie et leur clonogénicité, et peut supprimer leur dépendance visà-vis d'une interleukine [9, 21, 22] ; (3) chez la souris transgénique $b c l-2 / I g$, on observe une accumulation de cellules B centrofolliculaires dont la plupart ont quitté le cycle cellulaire et ont une durée de vie très augmen- 
Tableau I

MODIFICATIONS CARACTÉRISTIQUES DU PROCESSUS APOPTOTIQUE

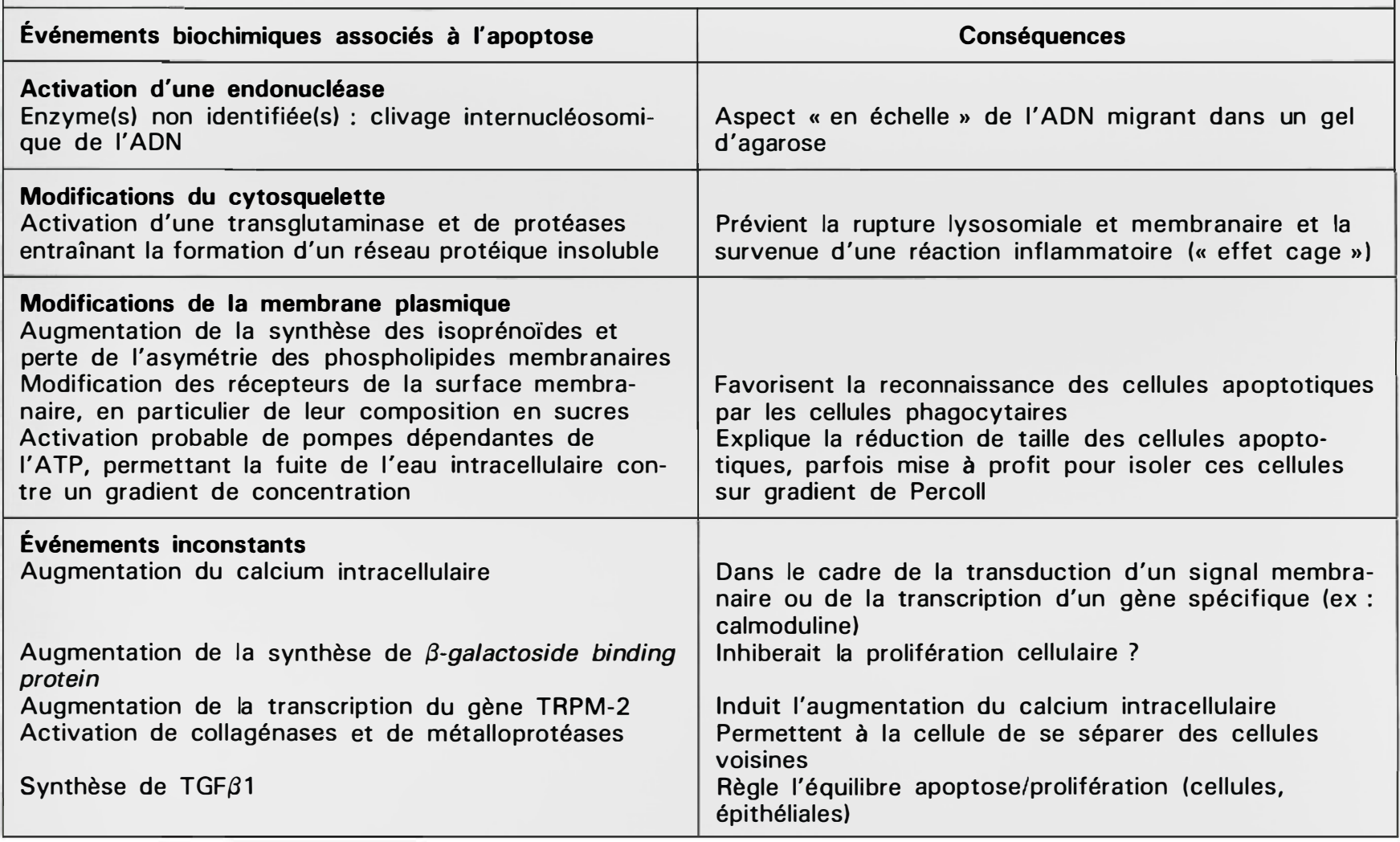

tée in vitro [9]. Le gène $b c l-2$ apparaît comme un nouveau type d'oncogène dont l'activation contribue au développement tumoral en inhibant la mort cellulaire. L'effet inhibiteur de bcl-2 n'est cependant pas universel. Son intervention dans la régulation de l'apoptose dépend de la cellule et/ou du signal induisant la mort cellulaire. A titre d'exemple, bcl-2 prolonge la survie des cellules dépendantes d'IL3, privées d'IL3, mais n'a aucun effet sur la survie de lignées dépendantes d'IL2 ou d'IL6, privées de leur facteur de croissance [9].

La protéine Bcl-2 est exprimée au niveau de la membrane interne des mitochondries [23]. Il a été suggéré qu'elle pourrait interférer avec une ou plusieurs fonctions mitochondriales telles que phosphorylation oxydative ou mouvements ioniques intracellulaires. Néanmoins, les mitochondries ne sont pas sa seule localisation comme cela a été récemment démontré avec $\mathrm{Bcl} 2$ exprimant son effet $m / s n^{\circ}$ 6-7 vol. 9, juin-juillet 93 anti-apoptotique dans une lignée cellulaire dépourvue de mitochondries $\left(\mathrm{m} / \mathrm{s} n^{\circ} 3\right.$, vol. 9, p. 328). Les mécanismes biochimiques de régulation de l'apoptose par la protéine Bcl-2 ne sont pas encore connus. A l'échelle tissulaire, la protéine $\mathrm{Bcl}-2$ participe à l'organisation du système immunitaire. Elle contrôle la sélection négative des cellules $B$ antigène-réactives et la production des lymphocytes B mémoires. Elle pourrait aussi jouer un rôle dans la sélection positive des lymphocytes $\mathrm{T}$ au niveau du thymus $\left(\mathrm{m} / \mathrm{s}\right.$ suppl. au $n^{\circ} 1$, vol. 5, p. 25 ; $n^{\circ} 10$, vol. 5, p. $788 ; n^{\circ} 4$, vol. 8, p. 392). En dehors du système immunitaire, Bcl-2 pourrait assurer la pérennité des cellules progénitrices ou effectrices (couche basale de l'épiderme ou des cryptes intestinales, cellules souches hématopoïétiques) au niveau desquelles elle est exprimée [9].

L'infection des lymphocytes B par le virus d'Epstein-Barr peut induire leur immortalisation. Parmi les gènes de ce virus, certains ont une structure voisine de celle de $b c l-2$, dont ils pourraient mimer les effets biologiques. Mais, surtout, l'une des huit protéines latentes du virus (LMP-1) peut activer $b c l-2$ dans les cellules infectées [24]. Ces interactions entre virus et gène $b c l-2$ pourraient expliquer la contribution de certains virus au développement de tumeurs malignes telles que le lymphome de Burkitt. Les observations faites chez les souris transgéniques $b c l-2 / I g$ ont également montré le rôle de $b c l-2$ dans l'oncogenèse des tumeurs et, d'une façon plus générale, l'importance des modifications génétiques interférant avec le programme de mort cellulaire. Après une phase d'hyperplasie lymphoïde polyclonale indolente, caractérisée par l'accumulation de lymphocytes $\mathrm{B}$ circulants à faible taux de prolifération mais à durée de vie augmentée, ces souris développent des lymphomes malins 


\section{RÉFÉRENCES}

27. Shi Y, Glynn JM, Guilbert IJ, Cotte TG, Bissonnette RP, Green DR. Role for c-myc in activation-induced apoptotic cell death in T cell hybridomas. Science 1992 ; $257: 212-4$

28. Colombel M, Olsson CA, Ng PY Buttyan R. Hormone-regulated apoptosis results from recntry of differentiated prostate cells onto a defective cell cycle. Cancer Res 1992 ; 52 : 4313-9.

29. Vaux DL, Cory S, Adams JM. Bcl-2 gene promotes hacmatopoictic cell surviva and cooperates with c-myc to immortalize pre-B cells. Nature 1988; $335: 440-4$

30. Bissonnette RP, Echeverri F, Mahboud A, Green DR. Apoptotic cell death induced by c-myc is inhibited by bcl-2. Nature 1992 ; 359 : $552-4$.

31. Fanidi A, Harrington EA, Evan GI Cooperative interaction between c-myc and bcl-2 protooncogenes. Nature $1992 ; 359$ 554-6.

32. Bond J, Dawson $\mathrm{T}$, Lemoine $\mathrm{N}$ Wynford-Thomas D. Effect of serum growth factors and phorbol ester on growth and survival of human thyroid epithelial cell expressing mutant ras. Mol Carcinogenesis 1992 ; 5 129-35

33. Yonish-Rouach E, Resnitzky D, Lotem J, Sachs L, Kimchi A, Oren M. Wild-type p53 induces apoptosis of mycloid leukacmic cells that is inhibited by interleukin-6. Nature $1991 ; 352: 345-7$

34. Shaw P, Bovey R, Tardy S, Sahli R, Sordat B, Costa J. Induction of apoptosis by wild-type p53 in a human colon tumor derived cell line. Proc Natl Acad Sci USA 1992 ; 89 : 4495-9.

35. Kuerbitz SJ, Plunkett BS, Walsh WV Kastan MB. Wild-type p53 is a cell cycle checkpoint determinant following irradiation Proc Natl Acad Sci USA 1992; 89 : 7491-5.

36. McConkey DJ, Orrenius S, Jondal M Cellular signalling in programmed cell death (apoptosis). Immunol Today $1990 ; 11$ : 120-1.

37. Itoh N, Yonchara S, Ishii A, Yonchara M, Mizushima SI, Sameshima M, Hase A Seto Y, Nagata S. The polypeptide encoded by the cDNA for human cell surface antigen Fas can mediate apoptosis. Cell $1991 ; 66: 233-43$

38. Trauth BC, Klas C, Peters AMJ Matzku S, Moller P, Falk W, Debatin KM, Krammer PH. Monoclonal antibody mediated tumor regression by induction of de haut grade de malignité [9]. Cette prolifération monoclonale secondaire résulte vraisemblablement de l'activation de nouveaux oncogènes, en particulier de $c-m y c$.

L'oncogène $c-m y c$, une des clés de la régulation de la prolifération cellulaire, intervient aussi dans la régulation de l'apoptose. Cette notion nouvelle et paradoxale a fait l'objet d'une récente analyse $\left(\mathrm{m} / \mathrm{s} n^{\circ} 6\right.$, vol. 8 , p. 586). L'apoptose est observée lorsqu'il existe un découplage entrc des niveaux élevés d'expression de $c^{-}$ myc et la disparition des signaux mitogéniques [10, 25, 26]. La protéine Myc agit vraisemblablement comme un facteur de transcription. Elle apparaît comme une composante de certaines voies d'induction de la mort cellulaire programmée : l'apoptose induite dans les cellules $\mathrm{T}$ immatures par activation du récepteur $\mathrm{T}$ pour l'antigène est inhibée par un oligonucléotide antisens correspondant à c-myc [27]. La voie d'activation de l'apoptose à laquelle participe c-myc est aussi une voie d'activation de la prolifération. Ainsi, chez lc rat, l'apoptose de la prostate ventrale déclenchée par la castration passe par une cascade d'activation génique $(c$ fos $>c-m y c>h s p-70)$ précédemment associée à la prolifération [28].

La protéine Myc est donc une étape commune aux voies d'activation de la mort et de la multiplication des cellules tumorales. Le corollaire est que la surexpression de Myc dans une cellule la protège de la transformation maligne en induisant son apoptose, à moins qu'un autre signal réoriente la voie d'activation de l'apoptose passant par Myc vers une progression à travers le cycle cellulaire et une stimulation de la prolifération. C'est ainsi que s'explique la coopération entre Myc et Bcl-2 dans la transformation maligne [29]. Il vient d'être montré que Bcl-2 prévenait la mort cellulaire induite par Myc sans abolir ses propriétés mitogéniques [30, 31]. Lorsque la mort cellulaire est inhibée, l'activation de nouveaux oncogènes à l'occasion de réarrangements chromosomiques ou de mutations géniques favorise la progression tumorale.

L'association Myc / Bcl-2 ne confère pas un phénotype classique de cellule transformée. L'apparition d'un tel phénotype semble nécessiter un événement supplémentaire tel quc l'activation d'un oncogène ras. L'activation de ras et ses interactions avec les systèmcs de transduction peuvent inhiber l'apoptose et stimuler la prolifération cellulaire. Ainsi, une lignée fibroblastique de rongeur transfectée avec c-myc ou c-Ha-ras devient tumorigène chez l'animal immunodéprimé. Le taux d'apoptose spontanée est élevé dans les tumeurs transfectées par c-myc mais très réduit dans les tumeurs transfectées par c-Ha-ras [26]. Cependant, l'activité prolifératrice de l'oncogène ras muté semble pouvoir être réorientée vers un signal de mort cellulaire par activation de la protéine kinase C par le tpA ([32] $\left(\mathrm{m} / \mathrm{s} n^{\circ} 6\right.$, vol. 8, p. 586). Cette observation récente suggère que plusieurs oncogènes pourraient participer à l'induction de la prolifération ou à l' induction de l'apoptose en fonction des signaux transmis à la cellule.

Les oncogènes $c$-myc et $c$-Ha-ras ne sont pas les seuls dont la dérégulation interfère avec l'apoptose. C'est aussi le cas du gène codant pour la phosphoprotéine nucléaire p53, un oncogène considéré comme suppresseur de tumeur. L'inactivation de la p53 par mutation ou délétion génique, ou par liaison à une protéine virale (antigène $\mathrm{T}$ de SV40) ou cellulaire (MDM2) est observée dans la plupart des tumeurs humaines. La p53 intervient dans le cycle cellulaire, en particulier dans la transition de G1 à S, en contrôlant la transcription de gènes spécifiques. La p53 intervient aussi dans l'induction de l'apoptose. L'équipe d'Oren a réintroduit une p53 dans des cellules leucémiques murines qui en étaient dépourvues. Le gènc transfecté pouvait s'exprimer soit sous la forme sauvage, soit sous une forme mutée en fonction de la température. La protéine sauvage, mais non la forme mutée, induit rapidement l'apoptose. Cet effet de la protéine sauvage peut être prévenu par l'IL6 [33]. Cette observation suggère que la p53 est nécessaire à l'induction du programme apoptotique. Sa disparition pourrait allonger la survie de cellules progénitrices normalement destinées à disparaître par apoptose en l'absence d'un signal de différenciation ou de prolifération. Les cellules ayant perdu la p53 progressent 
dans le cycle ccllulaire ct sc multiplient jusqu'à l'activation d'un nouvel oncogènc [28, 33, 34].

Sclon D. P. Lanc [11], la p53 agirait physiologiquement comme un des "gardicns" du génome cellulaire. Si l'ADN cst altéré, par excmple par des radiations, la p53 s'accumulc et bloque le cycle cellulairc en G1 pour permettre aux systèmes de réparation de fonctionner [35]. Si les lésions de l'ADN persistent ou si la ccllule ne reçoit pas les signaux de différenciation appropriés, la p53 pourrait induire l'apoptose. Si la p53 est absente, mutée ou inactivée, la cellule ne s'arrĉte pas pour réparer son génomc. Mutations et réarrangements chromosomiques s'accumulent ct conduisent à la sélection de clones malins. Lc haut nivcau d'ancuploïdie et de mutations obscrvé dans les tumeurs des souris n'exprimant pas la p53 ct l'instabilité génétique des fibroblastes des patients atteints de syndrome de Li Fraumeni pourrait s'expliquer par la suppression d'un programme apoptotique.

\section{Apoptose et récepteurs}

Chaque ccllule reçoit de multiples signaux (hormones, cytokines) par l'intermédiaire de réceptcurs spécifiques. Ces signaux peuvent induire l'cntrée de la cellule dans le cycle cellulaire ou son apoptose. A titre d'excmple, les glucocorticoïdes ou les ionophores du calcium induisent l'apoptose dans les thymocytes. Ce processus cst inhibé par activation de la protéine kinasc $\mathrm{C}$ par un ester de phorbol ou par l'IL1 [36]. L'altération d'un récepteur spécifiquc pourrait participer à l'apparition d'un clone malin en déséquilibrant cettc rclation entrc signaux inducteurs et répresseurs de l'apoptose et de la prolifération.

L'antigène Fas ou APO-1 cst une protéine transmembranaire exprimée notamment à la surface de certaines ccllules hématopoïétiques [37]. Fas / APO-1 appartient à une nouvelle famille de récepteurs partagcant des analogies de structure et de régulation avec les réccptcurs des TNF (tumor necrosis factor) et du NGF (nerve growth factor) ainsi que les antigènes CD40 ct OX40 exprimés respcctivcment à la surface des cellules B et T [37]. La liaison d'un anticorps spécifique antiAPO-1 à son réccpteur induit l'apoptose des cellules lymphoblastoïdes humaines SKW6.4 [38]. Cettc induction cst indépendantc de toute activation complémentaire. Un anticorps monoclonal peut donc être directement cytotoxique [23]. La transfection du gène humain codant pour Fas/ APO-1 dans des cellules de souris a permis de montrer que ce récepteur était bien lc médiateur du signal apoptotique. Chez les souris lpr caractérisées par le développement de syndromes lymphoprolifératifs et de manifestations auto-immunes, la mutation du domaine cytoplasmique de Fas / APO-1 bloque la transduction du signal induit par la liaison du réccpteur à un ligand [7]. Ces observations suggèrent que Fas/APO-1 pourrait être impliqué dans l'élimination des clones lymphocytaires $T$ autoréactifs au niveau du thymus et dans la régulation de la duréc de vic des lymphocytes B. Le système Fas / APO-1 agirait donc à l'inverse de Bcl-2.

Le fait que des anticorps dirigés contre le récepteur Fas / APO-1 puissent induire dircctement l'apoptose dans lcs cellules exprimant cc récepteur pourrait avoir des applications thérapeutiques in vivo. Leur utilisation a

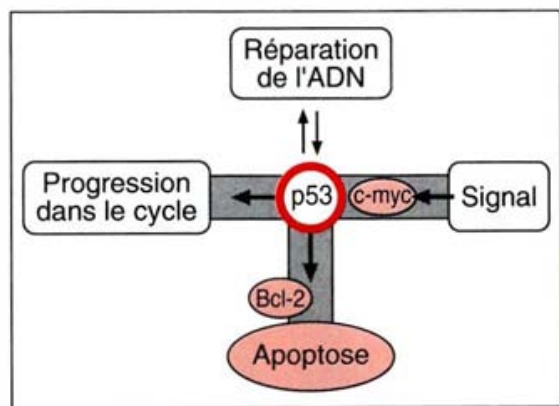

Figure 2. Rôle des oncogènes dans la régulation de la mort cellulaire programmée. L'activation de c-myc semble être commune à certaines voies d'activation de la prolifération et de I'apoptose. La p53 jouerait le rôle de point contrôle avant le passage de $G 1$ à $S$. Si l'ADN est altéré, la p53 inhiberait la progression dans le cycle cellulaire pour permettre à la cellule de réparer ces lésions. Si les réparations sont insuffisantes, la p53 orienterait le métabolisme cellulaire vers l'apoptose (d'après [11]). La protéine $\mathrm{Bcl}-2$, si elle est exprimée, peut inhiber le processus apoptotique. Dans ce cas, la cellule ne meurt ni ne prolifère. été envisagée dans le traitement des lymphomes de Burkitt APO-1 positifs. Cepcndant, toutes les ccllules exprimant APO-1 ne sont pas sensibles à l'activation de l'apoptose par un anticorps spécifique [39]. Les conséquences de l'activation d'APO-1 sont probablement modulécs par le programme génétique de la ccllule, en particulier par le niveau d'expression de $c-m y c$

En l'état actuel des connaissances, la stimulation de la prolifération et celle de l'apoptose scmblent passer par l'activation de gènes communs tels que $c$-fos et $c$-myc. Lc métabolisme cellulaire cst ensuite orienté vers l'un ou l'autre des deux processus au niveau de points-contrôle (figure 2). Les multiples signaux que reçoit la cellule règlent l'activité de ces pointscontrôle. L'un de ces points pourrait être la p53, dont on sait qu'elle cst impliquée dans la progression de $G_{1}$ à $\mathrm{S}$. Bcl-2, situé au départ de certaines voies d'activation de l'apoptose, scrait capable de bloquer la progression dans cette voic sans relancer la prolifération. Au-delà de ces pointscontrôlc, la transcription de gèncs spécifiques tels que TRPM-2 (apoptose) ou ornithine décarboxylase (prolifération) assurerait la progression du métabolisme cellulaire dans l'une ou l'autre voie [40].

\section{Apoptose et traitement anticancéreux}

La plupart des traitements utilisés dans lc domainc du cancer pcuvent induirc l'apoptose des ccllules tumorales, qu'il s'agisse de chimiothérapie, de radiothérapie ou d'hormonothérapie. Les mécanismes d'induction dépendent à la fois des cellules traitées et du traitement utilisé [41-44]. Il est possible de distinguer deux grandes voics d'activation de l'apoptose sous l'effet d'un traitement. Cette distinction est fondée sur la vitesse de survenuc de l'apoptose et l'éventuelle mise en jeu des gènes de régulation du cycle cellulaire.

La première voie d'induction est celle de la mort cellulaire programmée. Cette voie débutc avec l'activation d'oncogènes non spécifiques ( $c-f o s, c$ jun, $c-m y c)$. Au niveau des pointscontrôle, cette voie d'activation est orientée vers l'apoptose en réponse 


\section{RÉFÉRENCES}

39. Falk MH, Trauth BC, Debatin KM, et al. Expression of the APO-1 antigen in Burkitt lymphoma cell lines correlates with a shift towards a lymphoblastoid phenotype. Blood 1992 ; 79 : 3300-6.

40. Grassili E, Bettuzi S, Monti D, et al. Studies on the relationship between cell proliferation and cell death : opposite patterns of SGP-2 and ornithine decarboxylase mRNA accumulation in PHA-stimulated human lymphocytes. Biochem Biophys Res Commun 1991; 180 : 59-63.

41. Barry MA, Bchnke CA, Eastman A. Activation of programmed cell death (apoptosis) by cisplatin, other anticancer drugs, toxins and hyperthermia. Biochem Pharmacol $1990 ; 40$ : 2353-62.

42. Bertrand R, Kerrigan D, Sarang $M$, Pommier Y. Cell death induced by topoisomerase inhibitors. Role of calcium in mammalian cells. Biochem Pharmacol 1991 ; 42 : 77-85.

43. Bertrand R, Sarang M, Jenkin J, Kerrigan $\mathrm{D}$, Pommier $\mathrm{Y}$. Differential induction of secondary DNA fragmentation by topoisomerase II inhibitors in human tumor cell lines with amplified c-myc expression. Cancer Res 1991; 51 : 6280-5.

44. Martikainen P, Kyprianou N, Tucker RW, Isaacs JT. Programmed death of nonproliferating androgen-independent prostatic cancer cells. Cancer Res 1991; 51 : 4693-700.

45. Lotem J, Sachs L. Hematopoietic cytokines inhibit apoptosis induced by transforming growth factor $\beta 1$ and cancer chemotherapy compounds in myeloid leukemic cells. Blood 1992; 80 : 1750-7.

46. Baxter GD, Lavin MF. Specific phosphorylation in apoptosis induced by ionizing radiation and heat shock in human lymphoid tumor cell lines. J Immunol 1992 ; 148: 1949-54

47. Uckun FM, Tucl-Ahlgren L, Song CW, et al. Ionizing radiation stimulates unidentified tyrosine-specific protein kinases in human B-lymphocytes precursors, triggering apoptosis and clonogenic cell death. Proc Natl Acad Sci USA 1992 ; 89 : 9005-9.

48. Tanizawa A, Kubota $\mathrm{M}$, Hashimoto $\mathrm{H}$, Shimizu T, Takimoto T, Kitoh T, Akiyama Y, Mikawa H. VP-16-induced nucleotide pool changes and poly(ADP-ribose) synthesis : the role of $\mathrm{VP}-16$ in interphase death. aux signaux extracellulaires qui modulent leur activité (signaux inhibiteurs ou absence de signal). C'est le cas de l'apoptose induite par la privation hormonale dans des cellules prostatiques de rat $[28,44]$. Les molécules classiquement impliquées dans le cycle cellulaire, en particulier c-fos, c-myc puis la p53 sont activées. La cellule est cependant incapable de compléter le cycle cellulaire, probablement du fait de l'absence de signal mitogénique, et meurt par apoptose. Un arrêt en G1 ou surtout en G2 est une autre réponse observée dans des cellules tumorales traitées par un agent cytotoxique, qu'il s'agisse d'inhibiteurs des topo-isomérases (adriamycine, étoposide, téniposide, amsacrine, campthotécine), d'antimétabolites (ara-C, 5-fluoro-uracile, métothrexate), d'agents alkylants (cyclophosphamide, melphalan, cisplatine) ou d'inhibiteurs de la tubuline (vincristine, vinblastine) [18, 41-43]. Cet arrêt est interprété comme un moyen, pour la cellule traitée, de gagner du temps afin de réparer ses lésions avant de progresser plus avant dans le cycle cellulaire. En cas d'échec, la cellule entre en apoptose à distance de l'arrêt en $\mathrm{G}_{2}$ ou en $G_{1}$. Inversement, si les pointscontrôle tels que la p53 ont disparu au cours de la transformation maligne, la cellule ne s'arrête pas en cours de cycle pour réparer et accumule rapidement des lésions létales. La seconde voie d'induction est celle de la mort en interphase. L'apoptose est observée très rapidement dans les cellules tumorales traitées, moins d'une heure après le début du traitement. Le nombre de cellules apoptotiques est alors très supérieur au nombre de cellules dans une phase donnée du cycle cellulaire, suggérant que l'induction de l'apoptose est indépendante du cycle cellulaire. Tout se passe comme si la cellule était préprogrammée pour l'activation de la phase finale de l'apoptose, probablement l'activation d'une endonucléase. C'est le cas des cellules leucémiques humaines HL-60 traitées par des inhibiteurs des topoisomérases [43]. Les inhibiteurs de la synthèse des macromolécules n'ont aucune influence sur cette voie d'induction. Un changement du programme génétique de la cellule au cours d'un processus de différenciation [18], ou des signaux adressés à la cellule [45], peuvent modifier la sensiblité cellulaire à l'induction de l'apoptose.

Un même traitement peut induire l'apoptose selon l'une ou l'autre de ces voies en fonction de la cellule cible, de son degré de différenciation, des signaux qui lui sont adressés et de leur intensité. Ainsi l'apoptose induite par les glucocorticoïdes est prévenue par les inhibitcurs de synthèse des macromolécules dans les thymocytes immatures (mort cellulaire programmée) mais non dans les cellules de leucémie lymphoïde chronique (mort en interphase). Lorsque la p53 sauvage est transfectée dans les cellules HL-60, de faibles doses de radiation entraînent un arrêt en $\mathrm{G} 1$. Cet arrêt en G1 n'est pas observé dans les cellules HL-60 n'exprimant pas la p53 [35]. De ces observations, pourraient découler de nouvelles conceptions du traitement des tumeurs. D. P. Lane suggérait récemment que l'induction de la p53 par un agent non toxique dans les cellules normales devrait permettre de traiter les tumeurs avec de plus hautes doses de chimiothérapie ou de radiothérapie. L'efficacité thérapeutique serait augmentée sans accroître la toxicité [11]. Il est possible également que le niveau d'expression constitutive de $c$ myc influence la réponse apoptotique aux agents cytotoxiques [43].

La mort cellulaire induite par les cellules cytotoxiques est la seule qui semble indépendante de la cellule cible et de son programme génétique [16]. L'étude de ses mécanismes devrait permettre de connaître les éléments décisifs dans l'induction du processus apoptotique. Le jeu des phosphorylations / déphosphorylations pourrait être un de ces éléments décisifs [46, 47]. En inhibant la plupart des kinases intracellulaires, la staurosporine a un effet inducteur particulièrement puissant (Bertrand et al., soumis pour publication) tandis que l'acide okadaïque, un inhibiteur des phosphatases, peut induire ou prévenir l'apoptose selon les systèmes cellulaires. Il est possible que des agents anticancéreux, en cours de développement préclinique, agissant sur les systèmes de transduction cellulaire, se révèlent être des inducteurs spéci- 
fiques de l'apoptose dans les cellules malignes.

La capacité des cellules tumorales à entrer en apoptose devrait logiqucment être une des clés de la réponse au traitement des affections malignes chez l'homme. La modulation de l'apoptose pourrait avoir diverses implications : potentialiser l'activité antitumorale des agents cytotoxiques, réduire leurs effets indésirables (toxiques) en inhibant l'apoptose dans les cellules non tumorales, guider la recherche de nouveaux produits induisant sélectivement l'apoptose dans les cellules malignes. Les inhibiteurs de la synthèse des macromolécules ne préviennent pas la mort en interphase. En revanche, ils inhibent l'apoptose lorsque son induction passe par l'activation d'un programme génétique. Scules les molécules qui modifient la structure de la chromatine (polyamines, inhibiteurs de la poly[ADP]ribosylation) ou qui inhibent l'activitć nucléasique (zinc), retardent l'apparition de l'apoptose en interphase [48]

\section{Summary}

Apoptosis function in oncogenesis and cancer treatment

Spontaneous or induced-apoptosis regulates tumor growth. "Programmed cell death" appears to be dependent on several oncogenes. Some of these genes like $c-m y c$ have been previously involved in cell proliferation while others like $b c l-2$ were shown to be only apoptosis inhibitors. Cell metabolism is directed to cell death or proliferation depending on checkpoints involving molecules such as p53. The understanding of apoptosis mechanisms in tumor cells and apoptosis induction by various treatments offers new perspectives in malignant tumor prophylaxis and treatment.

\section{TIRÉS A PART}

E. Solary. 